\title{
Selektive Anticholinergika: stark wirksam an der Blase
}

- Um am Detrusormuskel die gewünschten Effekte zu erzielen, bedarf es bei Patienten mit überaktiver Blase vor allem einer Blockade der muskarinergen M3-Rezeptoren. Diese sind in der Blase reichhaltig vertreten. Selektiv an diesem Rezeptor angreifende Anticholinergika wie Darifenacin (Emselex ${ }^{\oplus}$ ) erweisen sich bei den Betroffenen als gleichermaßen effektiv wie nicht selektive Substanzen.
Weil die genannten M3-Rezeptoren in anderen Organen wie Herz und Hirn allenfalls spärlich vertreten sind, ist mit unerwünschten Effekten an diesen Organen kaum zu rechnen. Im Unterschied zu nicht selektiven Anticholinergika ist unter Gabe von Darifenacin weder mit einem Anstieg der Herzfrequenz noch mit einem verlängerten QT-Intervall zu rechnen, berichtete PD Dr. Friedhelm Späh, Krefeld.

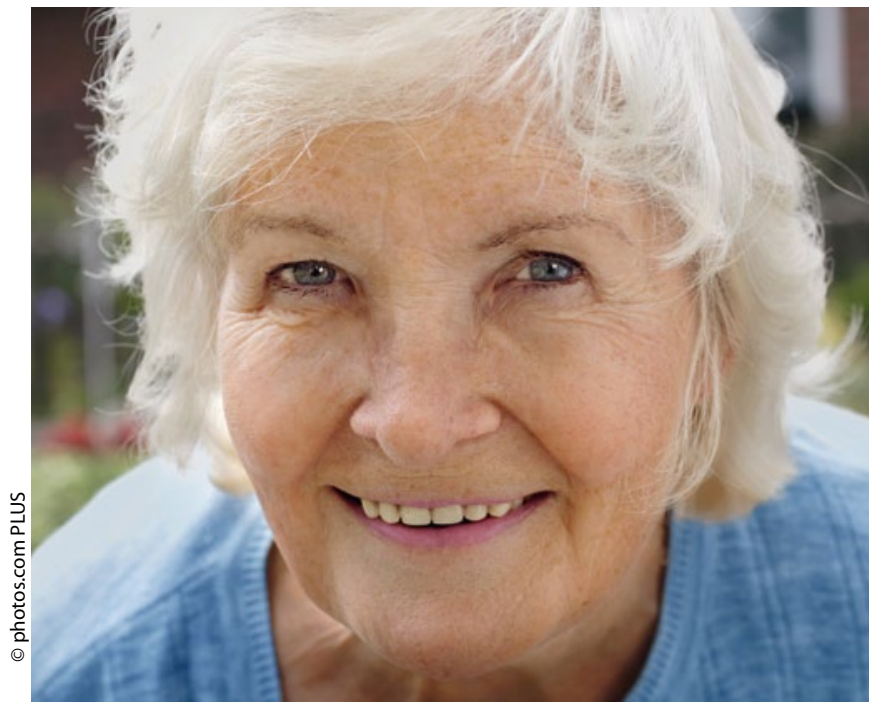

Bei Multimorbidität im höheren Lebensalter sollte auf potenzielle Interaktionen verschiedener Arzneimittel geachtet werden.
Auch mit unerwünschten Effekten auf die Hirnfunktion ist unter der Therapie mit einer M3-selektiven Substanz kaum zu rechnen, wie der Psychiater Dr. Klaus-Christian Steinwachs, Nürnberg, verdeutlichte. Mit psychometrischen Testverfahren konnte etwa gezeigt werden, dass das Kurzzeitgedächtnis unbeeinträchtigt bleibt.

Unerwünschte Effekte auf Herz und Hirn sollten besonders bei älteren Patienten vermieden werden, vor allem, weil diese Patienten aufgrund von Komorbiditäten häufig mit mehreren Medikamenten gleichzeitig behandelt werden. Ob ein Medikament aufgrund seines Nebenwirkungs- und/oder Interaktionsprofils ein im Alter potenziell ungünstiges Nutzen-Risiko-Profil hat, kann zum Beispiel unter www.priscus.net recherchiert werden. Bei der PRISCUS-Liste handelt es sich um ein von unabhängigen Experten erstelltes und vom Bundesministerium für Bildung und Forschung (BMBF) gefördertes Projekt.

Dr. Ludger Riem

Symposium „Polypharmazie - Eine Herausforderung in der OAB-Therapie", Köln, 4. November 2011

Veranstalter: Bayer HealthCare, Leverkusen

\section{LHRH-Therapie: Testosteronwerte regelmäßig überprüfen!}

- LHRH-Agonisten werden heute vor allem bei der Behandlung des lokal fortgeschrittenen oder metastasierten Prostatakarzinoms, bei Rezidiven nach Versagen einer kurativen Therapie und zur adjuvanten Therapie bei Risikopatienten in Kombination mit einer Operation oder Bestrahlung eingesetzt.

Neuere Untersuchungen zeigen, dass das Therapieziel bei fortgeschrittenem Prostatakarzinom kontinuierlich niedrige Testosteronspiegel $(<20 \mathrm{ng} / \mathrm{dl})$ sein sollten, um eine vorzeitige Krankheitsprogression zu verhindern. Die Praxis zeigt jedoch, dass sehr viele Ärzte keine regelmäßigen Testosteronmessungen durchführen und demnach die Effizienz der Therapie nicht kontrollieren.„Leider hat die Bestimmung der Testosteronwerte noch keine breite Akzeptanz gefunden. Dabei ist es die einzige Möglichkeit die Thera- pieeffizienz zu überprüfen", bemängelte Prof. Dietmar Molitor, Landau. Testosterondurchbrüche, Mini-Flares und ein hoher Testosteronnadir sind dagegen in der LHRH-Therapie immer noch sehr häufig zu finden. Bis zu $12,5 \%$ der Patienten unter Hormonsuppression mit Depotspritzen haben teilweise Testosteronwerte über $50 \mathrm{ng} / \mathrm{dl}$. Damit steigt das Risiko eines vorzeitigen Progresses.

„Dabei sind die Möglichkeiten, langfristig und kontinuierlich entsprechend niedrige Testosteronwerte zu erzielen, in der Praxis einfach durchzuführen, erläuterte Prof. Neal Shore, Myrtle Beach, USA. Das HistrelinJahresimplantat Vantas ${ }^{\circledR}$ erreicht diese Werte wie eine aktuelle Langzeitstudie zeigt. Im Rahmen der Zulassungsstudie wurden 138 Patienten mit fortgeschrittenem Prostatakarzinom eingeschlossen. Nach einem Be- handlungsjahr wurde innen angeboten, die Therapie fortzsetzen. So ergab sich ein Untersuchungszeitraum von bis zu 234 Wochen, in dem die Serum-Testosteronwerte dauerhaft bei weniger als $20 \mathrm{ng} / \mathrm{dl}$ (im Median 10,6-13,7) blieben. Dieser Effekt setzte bereits vier Wochen nach Studienbeginn ein. Das leicht und einfach einzusetzende und auch leicht wieder entfernbare Implantat bietet laut Neal ebenfalls Vorteile für die Lebensqualität der Patienten, da es Spritzen überflüssig macht.

Bettina Reich

Presse-Lunch „Aktuelles rund um die LHRHTherapie und Diagnostik beim Prostatakarzinom" im Rahmen des 63. DGU-Kongresses, Hamburg, 16. September 2011

Veranstalter: Orion, Hamburg 